$\begin{array}{lllllllll}\mathbf{A} & \mathbf{R} & \mathbf{T} & \mathbf{I} & \mathbf{C} & \mathbf{U} & \mathbf{L} & \mathbf{O} & \mathbf{S}\end{array}$ 


\title{
JUSTICIA PROCEDIMENTAL PURA Y TEORÍA DEL DERECHO ${ }^{1}$
}

\author{
Bruno Celano \\ Universidad de Palermo
}

\section{Teoría del Derecho}

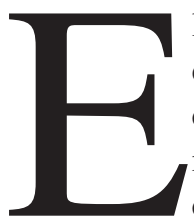

1 título de mi ponencia es 'Justicia procedimental pura y teoría del Derecho'. Adoptaré un particular paradigma teórico-jurídico que llamaré, en homenaje a Hans Kelsen, 'paradigma nomodinámico'. De acuerdo con el paradigma nomodinámico, la característica saliente para los fines de una comprensión adecuada del Derecho es el fenómeno de la autoridad: es decir, la institución de poderes de producción y aplicación de normas o actos jurídicos, mediante la especificación de las condiciones que individuos y grupos de individuos deben satisfacer para poder ejercitar tales funciones (es decir, mediante la constitución de órganos de producción y de aplicación del Derecho), así como de condiciones a las que la actividad de tales órganos debe conformarse para poder valer como ejercicio de las funciones en cuestión (es decir, la especificación de procedimientos apropiados a los fines de la producción o de la aplicación de normas o actos jurídicos). De acuerdo al paradigma nomodinámico, por decirlo brevemente, es característica saliente del Derecho la institución de poderes normativos: poderes, instituidos y disciplinados por normas, de producción o aplicación de normas.

\footnotetext{
${ }^{1}$ Una versión más amplia de este trabajo fue presentada al XXII Congreso nacional de la Sociedad italiana de filosofía jurídica y política ( «Giustizia e procedure. Dinamiche di legittimazione tra stato e società internazionale», Trieste, 27-30 de septiembre de 2000). Doy las gracias por sus comentarios y sus críticas a una versión precedente de este escrito, o por la discusión de algunos puntos problemáticos, a Mauro Barberis, Michelangelo Bovero, Pierluigi Chiassoni, Guido Corso, Pasquale De Sena, Enrico Diciotti, Jordi Ferrer, Ricardo Guastini, Giorgio Maniaci, Marco Mazzamuto, Tecla Mazzarese, José Juan Moreso, Giuseppe Nicolaci, Elena Pariotti, Anna Pintore, Aldo Schiavello, Isabel Trujillo y Francesco Viola. Debo añadir, sin embargo, que no he tratado aquí de replicar concluyentemente a algunas de las objeciones que juzgo más significativas. Hacerlo habría exigido un tratamiento sistemático de algunas opciones teóricas de fondo, y un desarrollo bastante más extenso de alguno de los argumentos adoptados aquí.
} 


\section{Justicia, procedimientos y Derecho}

¿Cómo se configura, desde el punto de vista de una teoría nomodinámica, la relación entre justicia y procedimientos?

Un primer aspecto de la respuesta a esta pregunta es completamente obvio: el Derecho es una institución que transforma, o al menos tiende a transformar, la solución de todo problema de justicia en una cuestión de ejecución de procedimientos predeterminados por parte de individuos predeterminados. Por ejemplo ¿deben satisfacerse las pretensiones de Tizio o bien las de Cayo, en conflicto con ellas? El enfoque específicamente jurídico de un problema de este tipo exige preguntarse: ¿quién es competente para juzgar la controversia surgida entre Tizio y Cayo, y según qué procedimiento debe hacerse el juicio con el fin de llegar a una solución que tenga carácter jurídico (es decir, que tenga consecuencias jurídicas: que incida sobre los derechos y las obligaciones jurídicas de quienes están sujetos al ordenamiento)?. En general, el Derecho tiende a transformar todo problema del tipo ¿¿Qué exige la justicia en la situación S?’ en un problema del tipo: ¿QQuién es competente para decidir, y en ejecución de qué procedimientos, qué cosa es debida a cada uno en S?'.

Va de suyo que esta transformación deja siempre un resto, un residuo. El Derecho tiende a llevarla a cabo, pero la transformación nunca es completa. No solamente porque el Derecho proporciona, además de especificaciones relativas a órganos y procedimientos de decisión, también criterios sustanciales de juicio, susceptibles de valoración desde el punto de vista de una teoría normativa de la justicia. Sino también porque se plantea en todo caso el problema de si la elección, por parte del Derecho, de un particular órgano en lugar de otro, o de un particular procedimiento en lugar de otro, es ella misma conforme a la justicia.

Ello no obstante, la tendencia, por parte del Derecho, a procedimentalizar, por así decirlo, cuestiones sustantivas me parece innegable. Esta constatación, así formulada, sigue siendo por completo genérica. Que las cuestiones jurídicas presenten siempre un aspecto procedimental, es, a fin de cuentas, una banalidad. Trataré ahora de especificar y desarrollar, en una dirección determinada, esta obviedad, deteniéndome en un aspecto particular de la relación entre justicia, procedimientos y Derecho. El aspecto en cuestión es la relación que se puede entender que se da entre Derecho y pensamiento jurídico, de un lado, y una particular articulación de la idea de justicia, de otro: la idea de una justicia que tenga ella misma carácter procedimental. 


\section{Justicia procedimental}

Como es sabido, en A Theory of Justice (1971) John Rawls introduce, en términos abstractos, la idea de una justicia «procedimental» y aporta una división de esta noción, distinguiendo tres formas fundamentales: justicia procedimental imperfecta, perfecta, y pura.

La división rawlsiana se fundamenta en dos parámetros ${ }^{2}$. El primero es la disponibilidad o no de un criterio de determinación de lo que es conforme a la justicia que sea independiente de, y preliminar respecto a, la ejecución del procedimiento especificado. El segundo parámetro es si existe o no la garantía de que el procedimiento especificado, si se sigue efectivamente, produzca el resultado conforme a la justicia.

En el caso de la justicia procedimental perfecta (ejemplificada, en $A$ Theory of Justice, por el problema de la división equitativa de un pastel) existe, en primer lugar, «un criterio independiente» de lo que sea conforme a la justicia, «un criterio definido separadamente de, y con carácter previo a, el procedimiento a seguir»; y, en segundo lugar, «es posible especificar un procedimiento que, con certeza, proporcione el resultado deseado». En el caso de la justicia procedimental imperfecta (ejemplificada por el proceso penal) por el contrario, «disponemos de un criterio independiente de lo que sea el resultado correcto, pero no hay ningún procedimiento practicable que conduzca con seguridad al mismo». Así pues, puede suceder que el procedimiento especificado sea seguido correctamente, y que, sin embargo, el mismo no produzca el resultado conforme a la justicia. En el caso, finalmente, de la justicia procedimental pura (ejemplificada por el juego de azar), «no hay ningún criterio independiente del resultado justo, pero hay un procedimiento correcto, o equitativo, tal que su resultado es, de la misma forma, correcto, o equitativo, sea el que sea». Una característica peculiar de la justicia procedimental pura es, observa Rawls, el hecho de que, para poder establecer qué estado de cosas es conforme a la justicia, el procedimiento especificado debe seguirse efectivamente; en efecto, no existe, en hipótesis, «ningún criterio independiente sobre la base del cual sea posible determinar que un cierto resultado es justo».

La noción de justicia procedimental pura promete, respecto a la noción de justicia procedimental imperfecta, una cierta ventaja ${ }^{3}$. Parece, en efecto,

2 Cfr., para lo que sigue, Rawls 1971, pp. 85-6, de donde provienen las citas.

${ }^{3}$ Rawls (1971, p. 85) afirma que «claramente, la justicia procedimental perfecta es rara, si no imposible, en cuestiones de un cierto interés práctico». Seguiré, en este punto, a Rawls, limitando mi análisis a las relaciones entre justicia procedimental pura e imperfecta (nada particularmente significativo se sigue de la extensión de las argumentaciones que se desarrollan en las páginas siguientes a la noción de justicia procedimental perfecta). 
garantizar una forma unívocamente definida, conceptualmente más económica, y menos controvertible (una forma por así decirlo mecánica, o automática) de afrontar problemas de justicia.

Por definición, en efecto, en el caso de un problema de justicia procedimental pura, no será necesario, una vez seguido el procedimiento relevante, indagar si el resultado al que de hecho ha conducido es o no conforme a la justicia. Esto no sucede, por el contrario, en el caso de la justicia procedimental imperfecta. En el caso de la justicia procedimental imperfecta, en efecto, una vez seguido el procedimiento especificado, sigue abierta en hipótesis una pregunta ulterior -una pregunta de más- respecto a las planteadas por un problema de justicia procedimiental pura: si la ejecución del procedimiento ha producido efectivamente el resultado conforme a la justicia, o no.

En este sentido, se puede afirmar que, en la justicia procedimental pura, el procedimiento relevante tiene carácter constitutivo de la conformidad a la justicia de aquello que constituye su resultado. La ejecución del procedimiento es, por así decirlo, infalible. Si, y sólo si, el procedimiento se sigue, el resultado es, necesariamente, conforme a la justicia; y es su efectiva ejecución lo que hace que aquello que constituye su resultado sea conforme a la justicia.

Bajo este aspecto, la justicia procedimental pura presenta una profunda afinidad estructural con el tipo de reglas denominadas por John R. Searle «reglas constitutivas». Las reglas constitutivas de Searle son reglas de la forma ' $\mathrm{X}$ tiene valor de (counts as) $\mathrm{Y}$ en el contexto C' (por ejemplo, 'quitarse el sombrero tiene valor de saludo en el grupo social G') que, dice Searle, crean (o definen), y también regulan, nuevas formas de comportamiento, las cuales no serían posibles en ausencia de las reglas mismas (esto es, formas de comportamiento cuyo concepto es lógicamente dependiente de la regla en cuestión). Las reglas constitutivas son contrapuestas por Searle a las reglas que llama «regulativas» (por ejemplo, "no se debe circular a velocidad superior a $120 \mathrm{~km}$ por hora'). Estas últimas, a diferencia de las reglas constitutivas, regulan formas de comportamiento cuyo concepto es lógicamente independiente de ellas ${ }^{4}$.

La afinidad entre la noción de regla constitutiva y la idea de justicia procedimental pura es, me parece, obvia. En el caso de una regla constitutiva (por ejemplo, la regla del juego del ajedrez que define el movimiento del alfil) la no conformidad del comportamiento efectivo con la regla no implica, por sí, que el comportamiento en cuestión constituya una trasgresión de la

\footnotetext{
${ }^{4}$ Searle, 1964 , pp. $111-4 ; 1965$, pp. 41-2; 1969, pp. 33-42, 50-3; 1995, pp. 27-8, 113-4.
} 
misma, o una violación; implica, más bien, que el comportamiento no es en absoluto una instancia (un token) del comportamiento (del type) definido por la regla. Así, por ejemplo, el traslado en L de un alfil en un tablero no es un mal movimiento; simplemente, no es en absoluto, en una partida de ajedrez, un movimiento. Del mismo modo, en un caso de justicia procedimental pura, qué valga como un estado de cosas conforme a la justicia está definido por el procedimiento relevante: el que X sea el resultado de la ejecución efectiva del procedimiento especificado es constitutivo de que sea conforme a la justicia.

La noción rawlsiana de justicia procedimental, considerada en abstracto, constituirá mi término de confrontación. Como es sabido, la teoría de la justicia de Rawls, así como la mayor parte de las teorías de la justicia postrawlsianas, pretende ser una teoría de la justicia global: su objeto es la estructura fundamental de la sociedad, considerada en su conjunto. Pero aquí no trataré, en primer lugar, de examinar si, y eventualmente en qué sentidos, una teoría de la justicia global pueda con aprovechamiento ser etiquetada como «procedimental»; ni, en segundo lugar, trataré de valorar, desde el punto de vista normativo (o ético), las perspectivas de éxito de una teoría procedimental de la justicia global. Lo que pretendo hacer es, más bien, poner a prueba la noción rawlsiana de justicia procedimental -en particular, la noción de justicia procedimental pura- desde el punto de vista de la teoría del Derecho; es decir, examinaré la relación que hay entre justicia procedimental pura y Derecho. Mi objetivo es, en otros términos, esclarecer cuál sea la suerte de la noción rawlsiana en el contexto de una concepción nomodinámica del Derecho.

\section{De la justicia procedimental pura a la justicia procedimental imperfecta}

Como he dicho poco antes, la justicia procedimental pura exige, a los fines de la determinación de qué sea conforme a la justicia, que el procedimiento especificado se siga efectivamente. Si, en cualquier ocasión, el procedimiento se ha seguido efectivamente es, al menos en parte, -en alguna medida- una cuestión de hecho; la respuesta correcta a este interrogante será, en línea de principio, accesible a una indagación de carácter teórico (es decir, dependerá del establecimiento de datos de hecho) ${ }^{5}$. Si no fuese así, en

\footnotetext{
${ }^{5}$ Con la expresión 'indagación teórica' me refiero aquí a una investigación que trate de dar respuesta a preguntas reconducibles a la forma: ¿Cómo están (estaban, estarán) las cosas (en el tiempo $t$ )?'. El adjetivo 'teórico' se contrapone aquí a 'práctico'. Tiene carácter práctico una indagación que trate de responder a preguntas reconducibles a la forma '¿Qué hacer (en $t)$ ?' (cfr. Celano 1994, pp. 678-90). Una repuesta apropiada a una pregunta de carácter teórico es (por definición) verdadera o falsa, correcta o no.
} 
efecto, el principio de justicia relevante habría de considerarse como mal formado, en cuanto que no apto para proporcionar ningún resultado determinado. Un procedimiento, en abstracto, se define como una serie de pasos, considerados como tipos abstractos; su ejecución consistirá en la instanciación de tales pasos. El problema de si una cierta serie de actos individuales constituye ejecución de un cierto procedimiento equivale, pues, al problema de si los actos en cuestión ejemplifican sus pasos (en la sucesión requerida). Se trata, en términos banales, de la cuestión de si un cierto individuo ejemplifica un tipo abstracto: de si cierto caso cae o no bajo una cierta regla.

Interrogantes de este tipo (si cierto caso cae o no bajo una regla) son, en el ámbito jurídico, de importancia crucial. Pero el Derecho -el modo de pensar específicamente jurídico- presenta, bajo este aspecto, una peculiaridad. Es decir, el modo de pensar jurídico está caracterizado por la constante conjunción de interrogantes de este tipo (si un cierto caso cae o no bajo una regla) con interrogantes de un tipo ulterior: si hay una instancia competente para juzgar, de acuerdo con modalidades específicas, cuál es la respuesta a dar a interrogantes del tipo anterior. En otros términos: en el ámbito jurídico, al interrogante ‘¿X es o no un caso de R?’ se añade constantemente el interrogante ulterior: ‘QQuién y según qué modalidades es competente para juzgar si X es o no un caso de R?'. Sólo la determinación por parte de una instancia competente, según modalidades especificadas, marca, desde el punto de vista jurídico, la diferencia: sólo una determinación hecha de esta forma tiene consecuencias jurídicas (es decir, incide sobre los derechos y las obligaciones de los individuos sujetos al ordenamiento).

Esto vale de forma absolutamente general. Y vale también, en consecuencia, con relación a la hipótesis de si es susceptible de aplicación, en el ámbito jurídico, la noción de justicia procedimental pura. Si, en un caso concreto, el procedimiento relevante se ha seguido o no es un problema del tipo: ‘¿X es o no un caso de R»?’. Quien se encuentre comprometido en una indagación normativa (en una indagación ética) puede, en línea de principio, determinar cuál sea la respuesta a esta pregunta; la suya será, con relación a este punto, una indagación de carácter teórico. Pero, desde el punto de vista jurídico, el problema será siempre: ‘Quién, y según qué modalidades, es competente para juzgar si se ha seguido el procedimiento relevante?'

Dicho brevemente: dado un caso de justicia procedimental pura, quien trata de responder a la pregunta (normativa, o ética) de si el resultado obtenido es o no conforme a la justicia, de un lado, y el jurista, de otro, acabarán necesariamente por plantearse problemas parcialmente distintos. El primero se preguntará si el procedimiento relevante se ha seguido efectiva- 
mente o no. El jurista, por su parte, se planteará, además de este primer interrogante, un interrogante ulterior: si al interrogante anterior se le ha proporcionado una respuesta (o si se le puede proporcionar una respuesta), con arreglo a modalidades de juicio establecidas, por un órgano competente. Entre la indagación normativa en materia de justicia, por un lado, y el modo de pensar jurídico, por otro, hay, en este aspecto, una discrepancia: a partir de un cierto punto, los respectivos recorridos inferenciales divergen entre sí. El jurista lleva a cabo, respecto al teórico de la justicia, una desviación.

¿Dónde conduce esta desviación? Para responder a este interrogante examinaré ahora un caso paradigmático del fenómeno sobre el cual he tratado de llamar la atención (esto es, la incidencia del modo de pensar típicamente jurídico en la consideración de interrogantes del tipo: ‘ ¿X es o no una instancia de R?': el problema de las normas, o de los actos, llamados 'irregulares' (leyes inconstitucionales, reglamentos ilegales, sentencias contra legem, etc.).

Simplificando al extremo, el problema es el siguiente. ${ }^{6}$ Desde el punto de vista de una teoría nomodinámica, una norma o un acto tienen existencia jurídica -puede decirse que son jurídicamente válidos, que son algo jurídico ( constituyen derecho ${ }^{7}$ - si, pero sólo si, satisfacen las condiciones es-

\footnotetext{
${ }^{6}$ En este parágrafo y en el siguiente (infra, 5) abordaré, de forma bastante sucinta y esquemática, el problema del carácter de las reglas que confieren poderes, o normas de competencia. Se trata, como es sabido, de uno de los temas cruciales de la teoría del Derecho (de la nomodinámica) contemporánea, ampliamente discutido, y con relación al cual se registran, en la literatura, puntos de vista profundamente divergentes. No trataré aquí de desentrañar este conjunto de opciones teóricas (si las llamadas normas de competencia, o al menos algunas de ellas, son normas permisivas, normas que imponen obligaciones, reglas constitutivas, meras definiciones, o más de una de estas cosas conjuntamente, o alguna otra cosa), ni proporcionaré una definición explícita de la clase de las normas de competencia. Me limitaré a asumir que las reglas de competencia tienen en algún sentido carácter definitorio, o constitutivo, de ser ciertos actos o normas actos o normas jurídicos, jurídicamente existentes o válidos. (Asunción, esta, que no es en absoluto incompatible con la tesis de que son fragmentos de normas condicionales en términos de obligación, o normas de ese tipo indirectamente formuladas; pero no es posible discutir aquí este punto: cfr. al respecto Celano 1999a, pp. 359-67). Espero que la argumentación presentada en el texto parezca al lector relativamente no controvertida, sea cual sea la concepción de las normas de competencia escogida (obviamente, dentro de ciertos límites). El problema de las normas o de los actos irregulares depende estrechamente, a su vez, de asunciones relativas a la naturaleza de las reglas de competencia; para una presentación particularmente clara del problema, y una exploración de las diversas posibilidades de solución, cfr. Moreso 1993.

7 Utilizo aquí como sinónimos los términos 'existencia' y 'validez' (referidos a normas y actos jurídicos). Los dos términos denotan, banalmente, el que una norma, o un acto, sean una norma o un acto jurídico (algo jurídico: algo que vale como Derecho). Se verá en el parágrafo siguiente (infra, 5) que la posibilidad de distinguir entre existencia y validez de normas y actos jurídicos, de acuerdo con líneas habituales en la literatura teórico-jurídica reciente no es, en relación con el tratamiento de nuestro problema, relevante.
} 
pecificadas por normas (ellas mismas normas jurídicas) que confieren poderes de producción y aplicación de normas: es decir, normas jurídicas que especifican quién (un órgano) y según qué modalidades (siguiendo qué procedimiento) es competente para producir una norma, o un acto, del tipo en cuestión ( a estas especificaciones pueden añadirse, y con frecuencia se añaden, vínculos referentes al contenido de las normas o de los actos producidos; de ahora en adelante, esta precisión se dará por sobreentendida). La falta de satisfacción de tales condiciones entraña, en línea de principio, que no alcance existencia (existencia jurídica) la norma, o el acto, en cuestión. El resultado de la actividad de una instancia no competente, o la falta de ejecución del procedimiento apropiado es, en línea de principio, jurídicamente inexistente, o inválido: no es en absoluto una norma, o un acto, jurídico.

Las reglas que confieren poderes de producción de normas o actos jurídicos presentan, pues, la misma característica conceptual que, como hemos visto, es propia de las reglas constitutivas y de las reglas de justicia procedimental pura. Las reglas constitutivas, se ha dicho, hacen posibles tipos de comportamiento o de entidades, que no existirían en su ausencia. De la misma forma, las reglas jurídicas sobre la producción de normas o de actos jurídicos hacen posibles tipos de comportamiento ( actividad de producción de normas o de actos jurídicos) que no serían posibles en su ausencia. En línea de principio, la conformidad de la actividad desarrollada por una cierta instancia con el procedimiento especificado por una regla de este tipo es constitutiva del ser, tal actividad, un caso de actividad de producción jurídica. La ejecución del procedimiento especificado por parte del órgano competente es, a su vez, constitutiva de ser el resultado jurídicamente existente.

Parece por ello que se puede afirmar la existencia de una profunda afinidad estructural entre problemas de justicia procedimental pura, por un lado, y problemas de validez, o de existencia, de actos o normas jurídicas (problemas del tipo: ‘'La norma N, o el acto A, es jurídicamente válida -es algo jurídico- o no?'), por otro. Las cosas, sin embargo, no son en absoluto tan simples.

En el ámbito jurídico, en efecto, la conformidad de un cierto comportamiento con las condiciones especificadas por reglas que confieren poderes de producción jurídica es únicamente en línea de principio constitutiva de que el resultado sea jurídicamente existente. En particular: no es del todo verdadero que la satisfacción de las condiciones relevantes sea condición necesaria de que cierta norma, o cierto acto, sea algo jurídico. Es, en efecto, un fenómeno innegable, y frecuente, que normas o actos sean tratados, por juristas y operadores jurídicos (en particular, funcionarios), como jurídicamente existentes aunque no satisfaga las condiciones de existencia (existencia jurídica, validez) establecidas por normas que confieren pode- 
res. No sólo: sucede a veces -bastante frecuentemente- que en casos de este género, el juicio sobre la efectiva conformidad o no de la norma, o del acto, a las condiciones relevantes sea él mismo deferido, por el Derecho, a un órgano predeterminado, habilitado para efectuar tal juicio de acuerdo con un procedimiento especificado. Sólo si se ha formulado por tal órgano, según el procedimiento apropiado, el juicio de no-conformidad del acto, o de la norma, en cuestión a los requisitos de validez relevantes tendrá consecuencias jurídicas. Un juicio (no importa aquí si verdadero o falso, correcto o no) que no constituya el resultado de la ejecución de un procedimiento apropiado por parte de un órgano competente no marcará, desde el punto de vista jurídico, ninguna diferencia.

En otros términos: desde el punto de vista jurídico, el problema de la validez de una norma, o de un acto, no es nunca -o mejor, no es nunca únicamente- si, efectivamente, la norma o el acto en cuestión ha sido producido por el órgano competente según el procedimiento apropiado. El problema es también, y sobre todo, si hay alguien que esté habilitado, por el Derecho mismo, para proporcionar, en ejecución de un determinado procedimiento, una respuesta a este interrogante, de forma tal que esta respuesta tenga consecuencias jurídicas. Dicho brevemente: en el ámbito jurídico, el problema de la regularidad de actos de producción jurídica es siempre el problema de la garantía de tal regularidad -de su garantía jurídica, o sea: de si el propio Derecho ofrece o no los medios necesarios para llegar a un juicio sobre la cuestión de la regularidad. El modo en el que puede suceder que el Derecho ofrezca tal garantía consiste, precisamente, en conferir un poder: en la especificación de un órgano habilitado para pronunciar el juicio de regularidad y de las modalidades a que tal órgano debe atenerse al formular tal juicio $^{8}$.

Pues bien: puede suceder que este ulterior juicio dé en el blanco, es decir, que sea juzgado inexistente un acto, o una norma, que efectivamente no constituye el resultado de la ejecución del procedimiento apropiado por parte del órgano competente, o existente un acto que constituye el resultado de un procedimiento de este género. Pero puede también suceder que el juicio en cuestión no de en el blanco: que sea juzgado inválido un acto en verdad válido, o válido un acto en verdad inválido. Ello no obstante, sólo un juicio oficial -un juicio hecho por el órgano competente, en ejecución del procedimiento apropiado- tendrá consecuencias jurídicas. Esto no quiere decir, bien entendido, que el Derecho sea lo que el órgano habilitado para el jui-

\footnotetext{
${ }^{8}$ Cfr. Kelsen 1942, trad. it. p.302: «no es (...) posible afirmar que un acto es nulo sin deber dar también una respuesta a la pregunta de quién es competente para establecer su nulidad».
} 
cio dice que es, punto y final. ${ }^{9}$ La corrección o no del juicio hecho por el órgano competente puede a su vez constituir el objeto de una indagación teórica. En la hipótesis de que sea establecida en su no-corrección, se deberá concluir que lo que el órgano competente ha juzgado que era Derecho no era, en verdad, Derecho (o viceversa). Sin embargo, el resultado de una indagación de este género no marcará, desde el punto de vista jurídico, ninguna diferencia.

La situación, es, pues, la siguiente. El Derecho proporciona, en hipótesis, un criterio independiente con arreglo al cual juzgar la validez de normas o actos que pretendan tener existencia jurídica. Tal criterio consiste en que sean, tales normas o actos, el resultado de la ejecución de procedimientos apropiados por parte de órganos competentes. Pero proporciona también (no necesariamente, y sólo dentro de ciertos límites, como veremos en breve) un procedimiento de juicio sobre la cuestión de si normas o actos son o no jurídicamente válidos, especificando cuál es el órgano habilitado para hacer tal juicio, y según qué modalidades debe hacerlo. Tal procedimiento, se espera, debería dar en el blanco (deberían ser jugados como inválidos, se espera, sólo actos o normas efectivamente inválidos y como válidos sólo actos o normas efectivamente válidos). Pero nada garantiza que esto tenga lugar. En suma: por un lado, se encuentra disponible un criterio de corrección independiente de, y previo a, la efectiva ejecución del procedimiento de juicio; de otro lado, no hay ninguna garantía de que el resultado de este último coincida con el establecido mediante la aplicación del criterio. Es decir, la situación es conceptualmente análoga a la ejemplificada por la justicia procedimental -no pura, sino- imperfecta: nos encontramos aquí frente a la conjunción de (1) disponibilidad de un criterio independiente de corrección y (2) no garantía de éxito del procedimiento ${ }^{10}$.

\footnotetext{
${ }^{9}$ Hart 1961, pp. 138-44.

${ }^{10}$ Una precisión. No pretendo sugerir que las condiciones de existencia jurídica, o validez, establecidas por reglas de competencia (condiciones relativas a órganos y procedimientos de producción, o al contenido de los actos o de las normas producidas) sean siempre, y necesariamente, tales que hagan que la cuestión de si una cierta norma, o un cierto acto, es o no conforme a tales condiciones, sea una cuestión de hecho, la respuesta a la cual sea accesible a una indagación teórica (es decir, susceptible de verdad o falsedad, correcta o no). La argumentación presentada en el texto no presupone esta asunción. Puede suceder que el Derecho especifique condiciones de existencia, o validez, tales que la respuesta a la cuestión de si un cierto acto, o una cierta norma, satisface las condiciones en cuestión no pueda calificarse ni como verdadera ni como falsa, ni como correcta ni como incorrecta (tales, esto es, que hagan que la respuesta a la cuestión de la regularidad esté al menos en parte indeterminada). Si esto sucede, y en la medida en que suceda, el Derecho deberá considerarse, en este aspecto, como indeterminado (no habrá una respuesta verdadera o falsa, correcta o incorrecta, a la pregunta de si un cierto acto, o una cierta norma, sea algo jurídico). La argumentación presentada en el texto no excluye esta posibilidad. Dicha argumentación, sin embargo, vale sólo con relación a la
} 
Podemos, en este momento, volver al problema que hemos dejado en suspenso. En el caso de la justicia procedimental pura, se decía, entre la indagación normativa en materia de justicia por un lado, y el modo de pensar jurídico, por otro, hay una discrepancia, una divergencia. Además del interrogante de si se ha seguido efectivamente el procedimiento relevante, el jurista se planteará, inevitablemente, un segundo interrogante: si hay un órgano habilitado por el Derecho para responder, en ejecución de un procedimiento determinado, al propio interrogante. ¿Dónde conduce esta desviación, esta discrepancia? El problema de las normas y de los actos irregulares sugiere una respuesta: hacia la justicia procedimental -no pura, sino imperfecta. Veamos por qué.

En el caso de la justicia procedimental pura, es necesario, a fin de determinar cuál sea el resultado conforme a la justicia, que el procedimiento se haya seguido efectivamente. Si el procedimiento se ha seguido efectivamente o no es, en hipótesis, una cuestión de hecho, susceptible de determinación. El problema específicamente jurídico, sin embargo, es: ¿quién, y según qué modalidades, está habilitado para controlar si lo que se presenta como ejecución del procedimiento relevante lo es efectivamente? En relación con esta segunda interrogante está disponible, en hipótesis, un criterio de corrección independiente del procedimiento de juicio cuestión, y no existe en segundo lugar, ninguna garantía de que la efectiva ejecución de este último de en el blanco. Así pues, el procedimiento cuya ejecución determina, en hipótesis, la solución de un problema de justicia procedimental pura aparece, en el ámbito jurídico, como el objeto posible de un procedimiento de juicio que presenta, a su vez, los rasgos de la justicia procedimental imperfecta. Conclusión: no se dan, desde el punto de vista jurídico, problemas de justicia que ejemplifiquen cabalmente la noción de justicia procedimental pura (no se dan, en el Derecho, problemas puros de justicia procedimental pura). El Derecho transforma toda cuestión de justicia procedimental pura en una cuestión de justicia procedimental imperfecta. Es decir, obtenemos, con relación a la hipótesis de que un problema de justicia procedimental pura se configure como un problema jurídico, la misma situación ejemplificada por el problema de las normas irregulares: disponibilidad de un criterio independiente de juicio y no garantía de éxito del procedimiento. El modo de pensar jurídico representa todo problema de justicia procedimental pura como el contenido de un problema de justicia procedimental imperfecta.

hipótesis de que el Derecho no sea, en este sentido, indeterminado, y en la medida en que no lo sea -vale, por decirlo así, por cuanto el Derecho es, en el sentido que se acaba de precisar, algo determinado. Hasta qué punto, en qué medida ('cuánto'), en estos aspectos, dentro de qué límites, un particular odenamiento jurídico (o un particular sector de un ordenamiento jurídico particular) sea, en el sentido precisado, determinado, es algo por completo contingente. 
En este aspecto, la noción de justicia procedimental pura es, desde el punto de vista de la teoría del Derecho, una noción irremediablemente ingenua: una construcción de moralistas. La razón de su ingenuidad reside en no tener en cuenta adecuadamente el hecho de que, en el ámbito jurídico, la interrogante de si un caso cae o no bajo una regla está siempre conjugada con la interrogante de si hay un órgano que tenga el poder de juzgar, según específicas modalidades, cuál sea la respuesta a dar al propio interrogante. La centralidad de esta pregunta ulterior es uno de los rasgos distintivos de una concepción nomodinámica del derecho.

\section{De la justicia procedimental imperfecta a la justicia procedimental pura}

La cosa no acaba aquí. Como acabamos de ver, hay un sentido en el cual el Derecho transforma todo problema de justicia procedimental pura en un problema de justicia procedimental imperfecta. Pero, como veremos seguidamente, en el Derecho también tiene lugar el fenómeno opuesto. Es decir, hay un sentido en el cual el Derecho transforma todo problema de justicia procedimental en un problema de justicia procedimental -no imperfecta, sino- pura.

Volvamos al problema de las normas y de los actos irregulares. Sucede a veces que normas o actos que, en realidad, no se han producido en cumplimiento de lo establecido por las normas que confieren poderes son tratados, por los juristas y operadores jurídicos, como actos o normas jurídicamente existentes (válidos), y que el juicio sobre su conformidad o no con las reglas relevantes se encuentra deferido, por el Derecho mismo, a órganos predeterminados como habilitados para hacer tal juicio en ejecución de procedimientos predeterminados. Esto no quiere decir, decíamos hace poco, que el Derecho sea lo que el órgano habilitado para el juicio dice que es, punto y final. Pero, una vez más, las cosas no son tan simples. En efecto: si bien la cuestión de si el acto o la norma es o no conforme a las reglas de competencia relevantes es, en hipótesis, una cuestión de hecho, la respuesta a la cual es en línea de principio accesible a una indagación teórica, sigue siendo siempre verdadero que una respuesta a este interrogante, aunque sea correcta, que no sea proporcionada por el órgano competente en ejecución del procedimiento apropiado, no marcará, desde el punto de vista jurídico, ninguna diferencia. Sólo un juicio oficial (un juicio hecho por el órgano competente en ejecución del procedimiento apropiado) tendrá consecuencias jurídicas. Puede, por ello, darse el caso siguiente: el acto, o la norma, es, en verdad, jurídicamente inexistente; pero el que lo sea no tiene, jurídicamente, ninguna consecuencia. Naturalmente, es posible que el Derecho confiera a un órgano ulterior, especificando un particular procedimien- 
to, el poder de juzgar si el juicio hecho por el órgano precedente es correcto o no, de forma tal que este segundo juicio tenga, a su vez, consecuencias jurídicas. Pero no es posible, aquí ir al infinito («es imposible proveer mediante reglas los instrumentos de corrección de las violaciones de toda regla» $)^{11}$. Así pues, puede suceder que actos o normas jurídicamente inexistentes (inválidos) sean, en última instancia, jurídicamente existentes (válidos), y tengan efectos jurídicos en conformidad con su contenido (y viceversa).

Ésta es, bien mirado, una contradicción. Una contradicción, sin embargo, que aparece como inevitable y que constituye una consecuencia necesaria de las condiciones que, desde el punto de vista de una teoría nomodinámica del derecho, definen el modo de pensar específicamente jurídico. La gravedad del problema, y la ineluctabilidad de la contradicción, pueden ilustrarse ulteriormente en un doble aspecto ${ }^{12}$.

(1) Alguien podría objetar que el problema de las normas irregulares, tal como se acaba de formular, es en realidad un pseudoproblema. Para darse cuenta de ello -prosigue la objeción- es suficiente con distinguir explícitamente ${ }^{13}$ dos diversas nociones de juridicidad (o sea, de conformidad a la reglas sobre la producción jurídica), una más débil y otra más exigente -las etiquetas son, aquí, irrelevantes: se puede distinguir entre mero 'vigor' (o 'vigencia') y 'validez' plena, o entre 'existencia' y 'validez', o entre simple 'aplicabilidad' y 'pertenencia' al sistema jurídico ${ }^{14}$, o entre 'validez 1' y 'validez 2', o entre 'existencia 1' y 'existencia 2'-, y reformular en los términos de esta distinción el (presunto) problema de las normas irregulares. Se dirá entonces (disipando, así, toda apariencia de contradicción) que sucede a veces que normas o actos meramente existentes, son tratados, por los juristas y operadores jurídicos como ('plenamente') válidos (pero la misma idea puede ser expresada distinguiendo, por ejemplo, entre 'vigor' y 'validez'), y que el Derecho confiere a un particular órgano el poder de juzgar, siguiendo un determinado procedimiento, acerca de su validez, de forma que tal juicio, y solamente él, tiene efectos jurídicos ${ }^{15}$.

\footnotetext{
${ }^{11}$ Hart 1961, p.139; cfr. Celano 1999a, pp. 70-2, 208-13.

12 Véase, para lo que sigue, Kelsen 1929, pp.1828-30, trad, it. pp. 162-5; 1942, trad. It. pp302-3. Cfr. también Guastini 1996, pp. 255-6, 262-5, 282-3; 1998, pp. 130-2, 135, 138-9, 147-8.

${ }^{13}$ Siguiendo una línea recientemente inaugurada, que yo sepa, por L. Ferrajoli $\left(1993^{\mathrm{a}}\right.$, pp. 148-9; 1993b, p. 465; cfr. también 1989, pp. 349-56, 915-8).

${ }^{14}$ Moreso 1997, pp. 158-9.

$15 \mathrm{O}$, alternativamente, que ciertas normas, aun no perteneciendo al sistema jurídico, son sin embargo aplicables en él (esto es, despliegan efectos jurídicos, en conformidad con su contenido), hasta cuando el relativo juicio de no pertenencia es efectuado por un cierto órgano, de acuerdo con un procedimiento determinado (cfr. Moreso 1997, pp. 158-9).
} 
Esta línea de disolución de la contradicción generada (sólo en apariencia, de acuerdo con esta forma de ver) por el problema de las normas irregulares parece, a primera vista, promisoria. Pero ¿verdaderamente es posible distinguir, de la forma requerida, dos diversas nociones (una más débil y la otra, por así decirlo, plena) de juridicidad (conformidad a reglas que confieren poderes) de forma que se disuelva el problema?

Naturalmente, nada impide la posibilidad de que reglas jurídicas establezcan que un acto, o una norma, haya de considerarse meramente existente (o meramente en vigor, etc.) si y sólo si satisface las condiciones C1...Cn, y sea por el contrario «válido» («plenamente válido») si y sólo si se satisfacen además también las condiciones Ca..Cx. Las condiciones C1....Cn constituirían, en esta hipótesis, un umbral mínimo superado el cual una (pretendida) norma, o un (pretendido) acto jurídico, constituye el objeto posible de un juicio oficial de validez; sólo un juicio tal tendrá, se asume, consecuencias jurídicas. Pero el problema es que, allí donde el Derecho lleve a cabo una especificación de este tipo, se planteará, desde el punto de vista jurídico, el problema de si hay un órgano competente para juzgar, en ejecución de un procedimiento determinado, si las condiciones en cuestión -las condiciones de existencia ( o 'vigor', etc.)- están satisfechas o no (es decir, si se ha alcanzado o no el umbral mínimo de juridicidad). En el Derecho, una especificación cualquiera de condiciones necesarias a fin de que se produzcan consecuencias jurídicas (por ejemplo la consecuencia consistente en que un cierto acto o una cierta norma sea tal que tenga necesidad, para no desarrollar ningún efecto jurídico, de un juicio oficial en tal sentido) abre, eo ipso, la posibilidad de que se plantee el interrogante: ¿quién, y según qué procedimiento, está habilitado para juzgar si las condiciones en cuestión están, en el caso en examen, satisfechas?

En otros términos. En el Derecho, se ha observado hace poco, puede suceder que actos o normas que no satisfacen las condiciones de validez relevantes sean tratados, por juristas y operadores jurídicos, como válidos, y que sólo un juicio oficial de irregularidad tenga consecuencias jurídicas. Y bien: de la misma forma, puede suceder en el Derecho que actos o normas que no satisfacen, en verdad, las condiciones de existencia relevantes sean tratados, por juristas y operadores jurídicos, como actos o normas jurídicamente existentes, y que sólo un juicio oficial de irregularidad tenga consecuencias jurídicas. Es éste, y no otro, el problema de las normas irregulares. El problema se plantea, de la misma forma, con relación a la mera existencia o al mero vigor de normas o actos jurídicos y con relación al problema de su validez plena. Es decir, se plantea de forma idéntica tanto si se distingue como si no se distingue entre varias nociones, progresivamente más exigentes, de juridicidad (validez, existencia jurídica; en general, conformidad 
a reglas de competencia) de actos o normas y sean cuales sean las etiquetas usadas para distinguir tales nociones. El problema es, en última instancia, el siguiente: ¿Es posible (no distinguiendo por el momento entre 'existencia' -'vigor', etc.- y 'validez' plena) que el Derecho especifique, en términos generales, un conjunto de condiciones tales que un acto o una norma que satisfaga tales condiciones, y sólo un acto o una norma que las satisfaga, sea algo jurídico, y produzca consecuencias jurídicas (incida sobre los derechos y obligaciones jurídicas de quienes están sujetos al ordenamiento); o, alternativamente (distinguiendo, ahora, entre mera 'existencia', o 'vigor', y 'validez' plena) condiciones tales que un acto una norma que satisfaga tales condiciones y, y sólo un acto o una norma que las satisfaga, constituya el objeto posible de un juicio de conformidad efectuado por un órgano determinado en ejecución de un procedimiento apropiado (es decir, alcance el umbral más allá del cual el juicio de no conformidad debe, para poder desarrollar efectos jurídicos, ser efectuado por un órgano determinado, en ejecución de un particular procedimiento)? El problema de las normas irregulares deriva de la circunstancia de que no parece que esto sea posible. La especificación, por obra del Derecho, de condiciones necesarias para la producción de una consecuencia jurídica cualquiera (por ejemplo de la necesidad de un juicio oficial a fin de que cierta norma, o cierto acto, no desarrolle consecuencias jurídicas) plantea, para la forma de pensar jurídica, la cuestión de si hay un órgano competente para determinar, en ejecución de un procedimiento determinado, la satisfacción o no de tales condiciones ${ }^{16}$.

\footnotetext{
${ }^{16}$ Adviértase: el surgimiento del problema no depende de la cuestión de si el conjunto de las condiciones de juridicidad (existencia jurídica, vigor, validez, etc.) de una norma, o de un acto, está definido o no de forma tal que incluya, además de condiciones relativas al órgano y al procedimiento de producción, también condiciones relativas a su contenido. El problema de las normas y de los actos irregulares se plantea de la misma manera en relación con ambos tipos de condiciones (cfr. Moreso 1993, pp. 82-3). Una teoría nomodinámica puede muy bien reconocer la posibilidad de que, en un sistema jurídico, entre las condiciones de validez de normas y actos jurídicos figuren también condiciones relativas a su contenido (y éste es el caso, por ejemplo, de la teoría pura del Derecho; cfr., por ejemplo, Kelsen 1934, pp. 74-6, 85-8, 91; 1945 , p. 124 ; 1960, pp. 12, 228, 230, 238, 241, 275). El problema es, sin embargo, que también en relación a este tipo de condiciones se plantea el problema de si hay un órgano competente para juzgar, en ejecución de un particular procedimiento, si ellas han sido satisfechas o no (de manera que el juicio hecho por tal órgano, y sólo él, tiene consecuencias jurídicas). Ciertamente, es posible distinguir entre 'existencia' (o 'vigor') de un lado, y 'validez' (validez 'plena'), del otro, definiendo la primera como mera conformidad de una norma, o un acto, a las condiciones, especificadas por el Derecho, relativas al órgano y al procedimiento de producción (o sólo a algunas de ellas), y la segunda como conformidad del acto o de la norma en cuestión, además de a las condiciones relativas al órgano y al procedimiento de producción, también a condiciones ulteriores, también ellas especificadas por el Derecho, relativas a su contenido. Se dirá entonces que puede suceder que una norma o un acto existente sea sin embargo inválido, puesto que, si bien satisface el conjunto de las condiciones, especificadas por
} 
(2) La misma dificulta aparece en el fenómeno que llamaré de la «evanescencia» de la distinción entre nulidad y anulabilidad (en una de las múltiples acepciones que estos dos términos asumen en el lenguaje jurídico).

En el Derecho, he dicho varias veces, sucede a veces que normas o actos jurídicamente inexistentes, o inválidos, son tratados, por juristas y operadores jurídicos, como jurídicamente existentes, o válidos ${ }^{17}$, y que el Derecho mismo confiere a determinados órganos el poder de juzgar, según un particular procedimiento, si las normas o los actos en cuestión son jurídicamente existentes o no (válidos o inválidos); sólo un juicio oficial tendrá consecuencias jurídicas. Pues bien: ¿cuándo, precisamente, nos encontramos frente a una situación de este tipo? Es decir: ¿en qué condiciones un acto, o una norma, que no satisface las condiciones de existencia (o validez) especificadas por reglas jurídicas no tiene ninguna necesidad, a fin de que sea jurídicamente inexistente (o inválido), de un juicio oficial en tal sentido?

Se sigue, de lo que se ha mostrado hasta ahora, que en el Derecho no es posible proporcionar, en términos generales, ninguna respuesta a este interrogante. Esto es, no es posible que el Derecho especifique, en términos generales, un conjunto de condiciones a fin de que un acto, o una norma, sea del todo inexistente, y no más bien un acto o una norma que es inexistente si, pero sólo si, el juicio de irregularidad relevante tiene carácter oficial. Un acto o una norma inexistente puede llamarse, banalmente, nulo; un acto o una norma cuyo carácter de inexistencia dependa de un juicio oficial en tal sentido puede llamarse, por el contrario, anulable. Pues bien: si, como se ha dicho, el Derecho no puede especificar ningún umbral mínimo de juridicidad, cuyo no alcance haga que un acto, o una norma, no constituya el objeto posible de un juicio oficial de regularidad, esto quiere decir que el Derecho no proporciona, ni puede proporcionar, ningún conjunto de condiciones sobre la base del cual distinguir entre actos o normas nulas y actos o nor-

el Derecho, relativas al órgano y al procedimiento de producción apropiados, no satisface el conjunto de las condiciones, también especificadas por el Derecho, relativas a su contenido; y que tal acto o norma puede correctamente, por esa razón, ser juzgado inválido, de manera tal que este juicio (si es hecho por el órgano competente, en ejecución del procedimiento apropiado) tenga consecuencias jurídicas. Esta distinción no está mal formada; pero no es adecuada para resolver el problema de las normas irregulares, ni constituye, me parece, un instrumento útil con vistas a su resolución.

${ }^{17}$ Nuevamente, en lo que sigue, 'existencia' y 'validez' (de normas y actos jurídicos) son usados como sinónimos (denotan ambos, indiferentemente, el que una norma o un acto sean una norma o un acto jurídico, en cuanto producido en conformidad con las reglas de competencia relevantes). Se acaba de mostrar en el texto que la posibilidad de definir los dos términos de forma que designen dos diversas nociones de juridicidad (conformidad a reglas de competencia) es, para nuestros fines, irrelevante. 
mas meramente anulables: no hay, desde el punto de vista jurídico, actos nulos, sino sólo actos anulables (según los casos, con diferentes consecuencias jurídicas $)^{18}$.

La conclusión es paradójica: la diferencia entre existencia e inexistencia jurídica (entre validez e invalidez) se sustrae, en última instancia, al Derecho. El criterio de discriminación entre actos o normas jurídicamente existentes, por un lado y actos o normas jurídicamente inexistentes, por el otro, no es ni puede ser, en última instancia, un criterio jurídico. En otros términos: en el Derecho, a la diferencia entre nulidad y anulabilidad, en línea de principio suficientemente clara, se desvanece (es, por así decirlo, evanescente). Esta diferencia no está representada, mediante especificación de criterios apropiados, dentro del propio Derecho. La cuestión de si un acto o una norma es tal que no tienen necesidad, para no existir, de un pronunciamiento oficial en tal sentido, es jurídicamente indecidible ${ }^{19}$.

Pero, si la diferencia relevante no depende del Derecho ¿de qué depende? No puedo proporcionar una respuesta satisfactoria a esta pregunta. Me limitaré a algún apunte en la dirección que me parece más promisoria.

En general, pueden denominarse hechos 'dependientes de la creencia' hechos que existen, que tienen lugar, sólo si se cree que existen, que tienen lugar: brevemente, si se da el caso de que $p$ sólo si se cree que se da el caso de que $p$. La dependencia de la creencia es una condición a primera vista paradójica. Sin embargo, muchos estarán dispuestos a conceder, me parece, que forman parte del mundo social un gran número de hechos que satisfacen esta condición. Así, por ejemplo, un cierto tipo de gesto es un saludo sólo si se juzga que es un saludo; algo es dinero sólo si se juzga que es dinero; un cierto conjunto de acontecimientos constituye un matrimonio sólo si se juzga que constituye un matrimonio; etc. Una teoría que atribuya a ciertos tipos de hechos sociales la característica de la dependencia de la creencia puede ser denominada, en sentido amplio, 'convencionalista' ${ }^{20}$.

${ }^{18}$ La diferencia entre nulidad y anulabilidad (en el sentido en el que estos términos son usados en el texto) acaba así por transformarse en la diferencia entre anulación (por parte del órgano competente, en ejecución del procedimiento apropiado) ex tunc y anulación ex nunc. Así entendida, la distinción entre nulidad y anulabilidad no coincide con la distinción discutida en el texto, y atañe, precisamente, a las diversas consecuencias jurídicas de los juicios de invalidez hechos por órganos competentes con arreglo a procedimientos apropiados.

19 'Indecidible' en el sentido siguiente: sobre la base de reglas jurídicas (reglas de competencia) es a veces posible responder negativamente a la interrogante formulada en el texto, pero no es nunca posible responder afirmativamente a la misma.

${ }^{20} \mathrm{La}$ tesis de que es característica propia de importantes (tipos de) hechos sociales sea la dependencia de la creencia es sostenida por J. R. Searle (1995, pp.1, 3, 13, 32-4, 52-3, 63, 69, 76, 88-9, 96, 191-4); cfr. también Lagerspetz 1995, pp. 6, 14, y passim. Para una definición más articulada y un análisis crítico de algunos aspectos del paradigma convencionalista cfr. 
Pues bien: ¿de qué depende de que un acto o una norma sea tal que no tenga necesidad, para no tener existencia jurídica, de un pronunciamiento oficial en tal sentido? La respuesta es, diría, la siguiente: que un cierto acto o una norma, a diferencia de otros, sea, en el Derecho, por completo irrelevante, y no constituya, por el contrario, el objeto posible de un juicio oficial de regularidad, es, en última instancia, un puro y simple hecho. Es un dato de hecho contingente, y nada más, que ciertos actos o normas, a diferencia de otros, sean tratados, por juristas y operadores jurídicos como jurídicamente irrelevantes por entero. Se trata, bien entendido, de un hecho de tipo peculiar. En última instancia, una norma o un acto es jurídicamente por entero inexistente si, de hecho, se juzga (se asume, se presume, se presupone, se da por descontado, se finge, etc.) etc. que lo es; y lo es, precisamente, en virtud de ser juzgado tal. El hecho en cuestión es, pues, un hecho dependiente de la creencia. Esto es, me parece que la consideración del problema de las normas irregulares lleva a la luz un punto en el cual el paradigma nomodinámico debe ceder el paso a, o de algún modo conjugarse con, un diverso paradigma teórico: el paradigma convencionalista.

Volvamos ahora a nuestro problema de fondo: ¿qué queda, en la teoría del Derecho, de la noción de justicia procedimental? El problema de las normas irregulares nos sitúa, como hemos visto, frente a la siguiente situación. En primer lugar, se encuentra disponible un criterio independiente de juicio sobre la corrección de la actividad relevante, y de su resultado; pero, desde el punto de vista jurídico, tal criterio no es en absoluto decisivo. En última instancia, sólo un juicio oficial de regularidad tendrá consecuencias jurídicas. En segundo lugar, se encuentra previsto un procedimiento cuyo éxito no está garantizado por nada: nada garantiza que el juicio oficial de regularidad de en el blanco. Pero, de otro lado, desde el punto de vista jurídico la posibilidad de fracaso está, en última instancia, excluida: en el Derecho, es jurídicamente existente, o inexistente, lo que el órgano competente, en ejecución del procedimiento especificado, juzga como tal - no importa si correctamente o no. Desde el punto de vista de una teoría nomodinámica del Derecho, pues, la situación está definida por los dos rasgos siguientes: no disponibilidad de un criterio independiente de corrección, y garantía de éxito del procedimiento de juicio. Esta situación es como se recordará, la situación ejemplificada por la noción de justicia procedimental (no imperfecta, sino) pura. Hay, pues, un sentido en el cual el Derecho

Celano 1997; 199b; 2000a ; 2000b. He probado una utilización parcial del paradigma convencionalista, limitado al análisis de la costumbre como fuente de producción jurídica en Celano 1995 y 1996. 
transforma todo problema de justicia procedimental imperfecta en un problema de justicia procedimental pura.

\section{Conclusiones}

Parecen emerger dos conclusiones de lo que se ha mostrado hasta ahora. En primer lugar, todo caso de justicia procedimental, aun si pura, se configura, desde el punto de vista jurídico, como un caso de justicia procedimental imperfecta. En segundo lugar, todo caso de justicia procedimental, aún si imperfecta, se configura, desde el punto de vista jurídico, como un caso de justicia procedimental pura.

Estas dos conclusiones están, a primera vista, en conflicto. La moraleja que de las mismas se puede extraer es, me parece, la siguiente.

(1) La distinción entre justicia procedimental pura y justicia procedimental imperfecta está, desde el punto de vista de la teoría del Derecho, mal construida (y la argumentación implica, banalmente, también a la noción de justicia procedimental perfecta). Las dos (presuntas) formas de justicia procedimental colapsan la una en la otra.

(2) Una teoría normativa de la justicia que se funde sobre la noción de justicia procedimental pura es, desde el punto de vista de la teoría del Derecho, irremediablemente ingenua. La razón de su ingenuidad reside en no tener en cuenta adecuadamente la necesidad de que, en sede jurídica, el juicio sobre la ejecución de un procedimiento cualquiera está sometido a una mediación autoritativa institucionalizada. Teorías de este tipo, en suma, no tienen en cuenta adecuadamente el principio nomodinámico.

(3) En el Derecho, puede suceder que sea tratado como algo jurídico algo que no tiene carácter jurídico, y viceversa. Hasta que la discrepancia no sea establecida por un órgano competente (en el supuesto de que haya uno), en ejecución de un procedimiento especificado (admitido, una vez más, que haya uno), la norma o el acto en cuestión producirán consecuencias jurídicas. Que el juicio de regularidad se difiera a un órgano ulterior implica que la misma posibilidad de discrepancia se presenta en relación con el juicio hecho por tal órgano. Puesto que no es posible seguir hasta el infinito, puede suceder que, en última instancia, tenga existencia jurídica un acto o una norma que no tiene existencia jurídica (y viceversa).

(4) El modo de pensar jurídico introduce, en relación con la aplicación de cualquier regla, una indecidibilidad de principio: qué acto, o qué norma, sea nulo, en vez de meramente anulable, depende de hechos, no del propio Derecho. Está indecidibilidad es un síntoma de la dependencia de la creencia que es propia de todos los hechos jurídicos. Una teoría adecuada de los hechos jurídicos, y una clarificación satisfactoria del papel que la ejecución de procedimientos cumple en el ámbito jurídico, deben ser capaces de pro- 
porcionar una solución al problema de las normas y de los actos irregulares. Una teoría de este tipo deberá, verosímilmente, representar a la existencia de normas, actos y hechos jurídicos como algo convencional.

(Traducción de Juan Ruiz Manero)

\section{Referencias bibliográficas}

Celano, B. 1994 Dialettica della giustificazione pratica. Saggio sulla legge di Hume, Giappichelli, Torino.

Celano, B. 1995b Consuetudine, convenzioni, en P. Commanducci, R. Guastini (eds.), Analisi e diritto 1995, Giappichelli, Torino. Trad. castellana Costumbres, convenciones, en Celano $2000 \mathrm{c}$.

Celano, B. 1996 Consuetudine e norme sulla produzione di norme, en P. Commanducci, R. Guastini (eds.), Struttutura e dinamica dei sistemi giuridici, Gappichelli, Torino. Trad. Castellana Costumbre y normas sobre la producción de normas en Celano 2000c.

Celano, B 1997 Fatti istituzionali: la teoria di J. R. Searle, en P. Commanducci, R. Guastini (eds.) Analisi e diritto 1997, Giappichelli, Torino.

Celano, B. 1999a La teoria del diritto di Hans Kelsen. Una introduzione critica, Il Mulino, Bologna.

Celano, B. 1999b Institutional Facts: The Realist Approach, ponencia presentada a la «Alf Ross Conference», Copenhagen, 11-12 junio 1999.

CElano, B. 2000a Fatti istituzionali e fatti convenzionali, «Filosofia e questini pubbliche», 5 .

Celano, B. 2000b Kelsen's Concept of the Authority of Law, «Law and Philosophy», 19.

Celano, B. 2000c Dos estudios sobre la costumbre, Fontamara, México.

FERRAJOLI, L. 1989 Diritto e ragione. Teoria del garantismo penale, Laterza, RomaBari 1990 ( $2^{\mathrm{a}}$ ed.).

FERRAJOLI, L. 1993ª Il diritto come sistema di garanzie, «Ragion pratica», 1.

Ferrajoli, L. 1993b Note critiche ed autocritiche intorno alla discussione su «Diritto e Ragione», en L. Gianformaggio (ed.), Le ragioni del garantismo. Discutendo con Luigi Ferrajoli, Giappichelli, Torino.

Guastini, R. 1996 Distinguendo. Studi di teoria e metateoría del diritto, Giappichelli, Torino.

Guastini, R. 1998 Teoria e dogmatica delle fonti, Giuffrè, Milano.

HART, H.L.A. 1961 The Concept of Law, Clarendon Press, Oxford.

Kelsen, H. 1929 Wesen und Entwicklung der Staatsgerichtsbarkeit, reimpreso en H. Kelsen, A. J. Merkl, A. Verdross, Die Wiener Rechtstheoristische Schule, hrsg. Von H. Klecatsky, R. Marcic, H. Schambeck, Europa Verlag, Wien, 1968. Trad. italiana (de la ed. francesa) La garanzia giurisdizionale della costituzione ( $L a$ Giustizia costituzionale) en H. Kelsen, La giustizia costituzionale, Giuffrè, Milano. 
Kelsen, H. 1945 General Theory of Law and State, Harvard U.P., Cambridge (Mass.).

Kelsen, H. Reine Rechtslehre, segunda edición, Verlag F. Deuticke, Wien.

Lagerspetz, E. 1995 The Opposite Mirrors. A Conventionalist Theory of Institutions, Kluwer, Dordrecht.

MoReSo, J. J 1993 Sobre normas inconstitucionales, reimpreso en J. J. Moreso, Normas jurídicas y estructura del Derecho, Fontamara, México, 1997.

MoRESo, J. J. 1997 La indeterminación del Derecho y la interpretación de la Constitución, Centro de Estudios Políticos y Constitucionales, Madrid.

RAWLS, 1971 A Theory of Justice, Oxford U. P., Oxford.

SEARLE, J. R. 1964 How to Derive 'Ought' from 'Is', reimpreso en P. Foot (ed.), Theories of Ethics, Oxford U. P., Oxford, 1971.

SEARLE, J. R. 1965 What Is a Speech Act?, reimpreso en J. R. Searle (ed.), The Philosophy of Language, Oxford U. P., Oxford 1971.

SeArle, J. R. 1969 Speech Acts. An Essay in the Philosophy of Language, Cambridge U. P., Cambridge.

Searle, J. R. 1995 The Construction of Social Reality, Penguin, Harmondsworth. 
$\triangle \quad$ DOXA 24 (2001) 